\title{
Influence of Pump Laser Phase and Amplitude Distortions on Terahertz Generation Efficiency
}

\author{
Lu Wang ${ }^{1,3}$, Arya Fallahi ${ }^{1}$, Koustuban Ravi ${ }^{1,2}$ and Franz X. Kärtner ${ }^{1,3}$ \\ ${ }^{1}$ Center for Free-Electron Laser Science, DESY, Notkestrasse 85, 22607 Hamburg, Germany \\ ${ }^{2}$ Massachusetts Institute of Technology, Cambridge, MA, 02139 USA \\ ${ }^{3}$ Department of Physics, University of Hamburg, Jungiusstrasse 9, 20355 Hamburg, Germany \\ Email: lu.wang@desy.de
}

\begin{abstract}
We analyze the impact of phase front and intensity profile distortions of the pump laser on the generation of narrowband terahertz radiation by difference frequency generation in periodically poled Lithium Niobate (PPLN). A code employing finite difference method for beam propagation and nonlinear interaction in cylindrical symmetric system is developed. The simulation accounts for difference frequency generation, selffocusing, self-phase modulation, diffraction, dispersion and terahertz absorption. We find that terahertz generation in PPLN crystals shows negligible sensitivity to spatial intensity modulation. In contrast, phase front modulations may drastically reduce the terahertz generation efficiency. Thus, care must be taken to avoid large phase distortions in high power laser beams for laserdriven terahertz sources.
\end{abstract}

\section{INTRODUCTION}

Due to the finite damage threshold of Lithium Niobate, high absorption of the material at terahertz frequencies and low single photon conversion efficiency (Manley-Rowe limit), generation of terahertz radiation with greater than percent level efficiency is extremely challenging. High terahertz generation efficiency requires high energy input pump lasers, where distortions in both intensity profile and phase front are unavoidable in current technologies. Our 2-D model brings the possibility of examining the influence of transverse variations of the pump pulse on terahertz generation. This cannot be captured by 1-D calculations. The presented results are based on the interaction between two narrow-band spectral lines separated by the desired terahertz frequency $(0.3 \mathrm{THz})[1]$. We assume a pump super-Gaussian pulse spatial profile of order $M=5$ and beam waist $\sigma=5 \mathrm{~mm}$. The distribution of the optical pump electric field in transverse dimension is defined as $E(r) \propto \exp ^{-\left(r^{2} / 2 \sigma^{2}\right)^{M}}$, where $r$ is the transverse dimension in the cylindrical coordinates.

\section{RESUltS}

In order to examine the influence of pump laser phase and amplitude distortions on terahertz generation, three cases are studied.

I. flat spatial intensity profiles with one of the pump spectral lines' phase-front being sinusoidally modulated from $-\pi$ to $\pi$ (Fig. 1(a1,a2)).

II. flat phase fronts with one of the spectral lines having a $\sim 10 \%$ sinusoidal intensity modulation (Fig. 1 (b1,b2)).
III. both spectral lines have flat intensity profiles and phase fronts (Fig. 1 (c1,c2)).

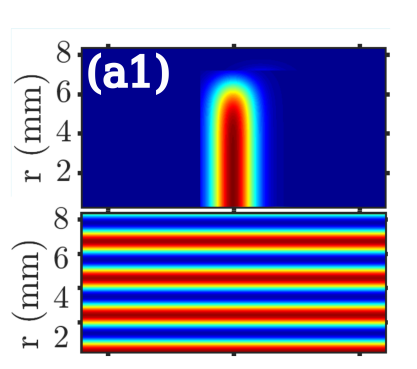

$291.25 \quad 291.26291 .27291 .55 \quad 291.56 \quad 291.57$

Freq $(\mathrm{THz})$
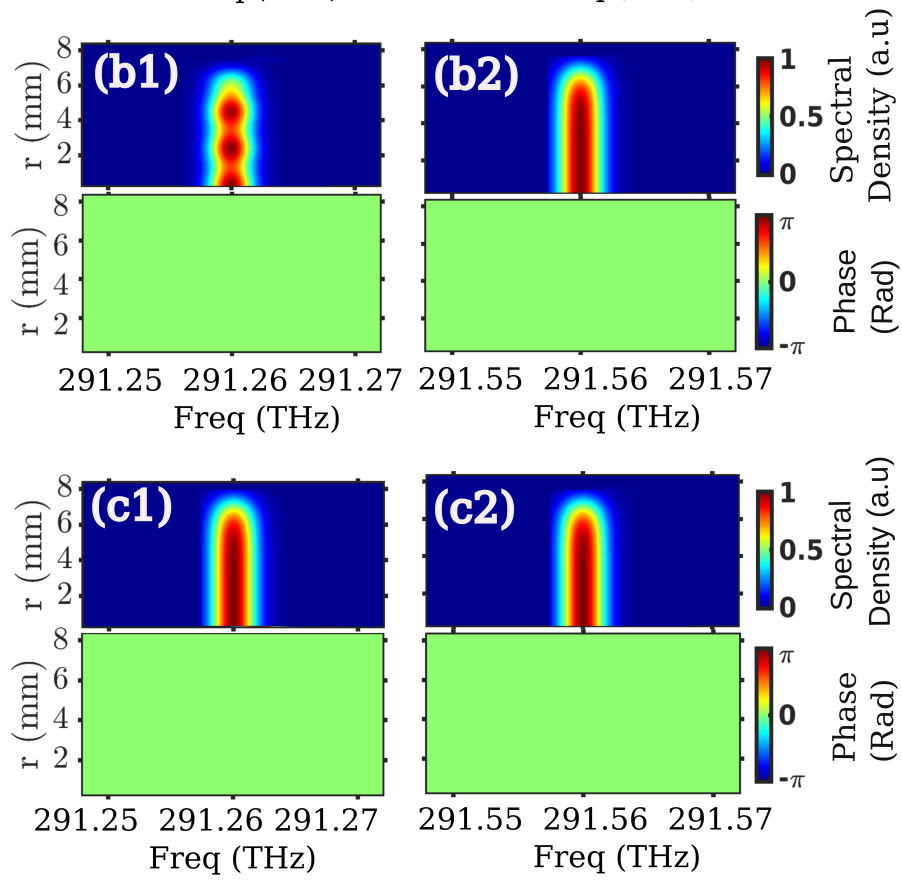

Fig. 1: (a1,a2), (b1,b2) and $(\mathrm{c} 1, \mathrm{c} 2)$ show the spectral density (first row) and phase front (second row) of the input pump spectral lines for case I, II and III, respectively.

The simulation results are depicted in Fig. 2. It can be seen in Fig. 2(a,c) that the $\sim 10 \%$ intensity profile modulation has negligible influence on the terahertz generation efficiency, whereas a distortion in phase front by a wavelength, not 
unusual for high power laser beams, strongly reduces the conversion efficiency.
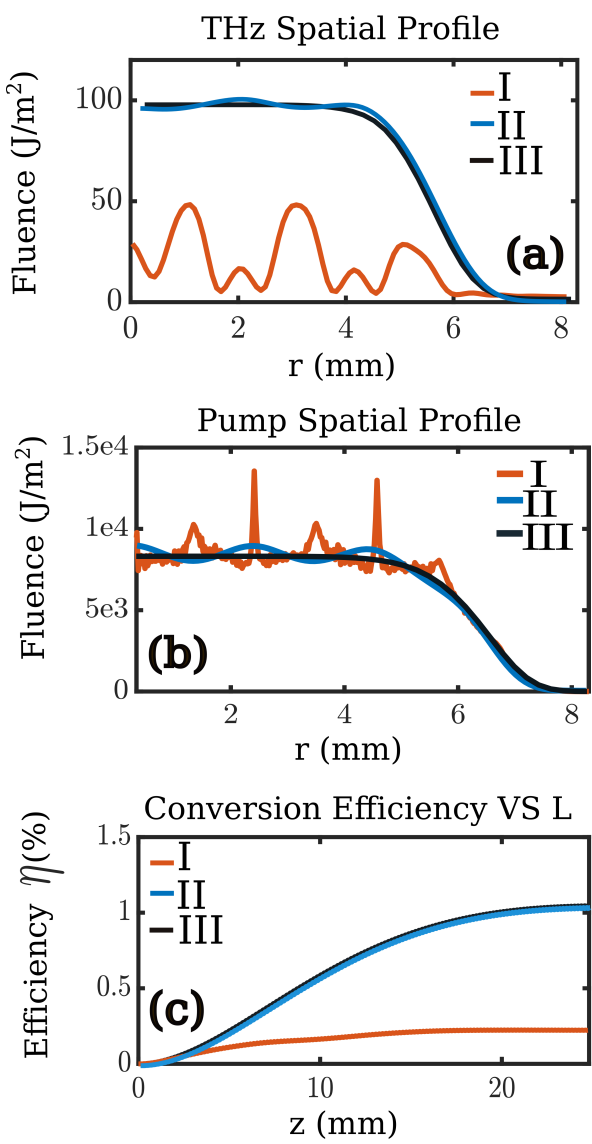

Fig. 2: (a) shows the resulting terahertz spatial profiles after $2.5 \mathrm{~cm}$ propagation length. (b) represents the optical pump spatial profiles after $2.5 \mathrm{~cm}$ propagation length. (c) shows the efficiency versus propagation length.

This is due to the nature of the difference frequency generation process, which transfers the phase difference between the two frequency lines of the optical pump to the terahertz beam at a thousand times longer wavelength. In turn, the modulated terahertz phase front causes dramatic diffraction, leading to destructive interference. Consequently, the terahertz conversion efficiency is greatly reduced and a strongly spatially modulated terahertz beam appears.

In Fig 2(b), the peaks of the optical pump spatial profile for case I is caused by self-focusing. Since the difference frequency generation process creates a terahertz pulse with the phase difference of the pump spectral lines, the new pump frequency lines generated by the cascading process carry different phases. The later generated cascaded lines inherit multiple times of the original phase difference, forming an enormously curved phase front. Thus, strong self-focusing occurs.

In addition, we find that if both of the pump lines are identically modulated in phase, the terahertz generation process is not degraded.

This work thus informs measures to be taken to circumvent phase induced efficiency deterioration for high energy terahertz generation.

\section{REFERENCES}

[1] K. Ravi, D.N. Schimpf, and F. X. Kärtner, "Pulse sequence for efficient multi-cycle terahertz generation in periodically poled lithium niobate," Opt. Express vol. 24, no.25,pp.582-25,607, 2016 